Ann. Zootech., I976, 25 (4), 543-549.

\title{
UTILISATION DES PRINCIPAUX ÉLÉMENTS MINÉRAUX DU MAIS ENSILÉ PAR LE MOUTON EN CROISSANCE
}

\author{
I. GUEGUEN et Michelle I)URANI) \\ avec la collaboration technique de Monique Allez, Pierrette Cams's et J. Gauther
}

Station de Recherches de Nutrition. Centre national de: Recherches zootechniques, I. N. R. A., 783.50 Jouy en Josas

\section{RÉSUMÉ}

Des bilans minéraux (l', Ca, Mg, $K, \mathrm{Na}$ ) ont été effectués sur 6 agneaux de $28 \mathrm{~kg}$ consommant seulement de l'ensilage de maîs $\left(3,8 \mathrm{~g}\right.$ cle Ca et $2, \mathrm{I}$ g de $\mathrm{I}^{\prime}$ par $\mathrm{kg}$ de matière sèche) enrichi en urée mais sans complément minéral. Les pertes fécales endogènes de phosphore ont été déterminées par la méthode de dilution isotopique.

Le coefficient d'utilisation digestive réelle du phosphore a atteint $7^{\circ} \mathrm{p}$. roo tandis que $34 \mathrm{p}$. roo seulement clu calcium ont été absorbés (tabl, I).

Les quantités journalières retenues étaient de 0,39 g pour le calcium et de $0,20 \mathrm{~g}$ pour le phosphore, valeurs environ trois fois trop faibles pour une croissance de i fo gr par jour. De mème, l'apport cle solium a été insuffisant.

Les pertes fécales endogènes de phosphore ont représenté $66 \mathrm{p}$. Ioo des pertes fécales totales, soit $36 \mathrm{mg}$ par $\mathrm{kg}$ de poids vif et par jour. Cette dernière valeur est normale pour un régime à base de fourrage grossier, puisque l'élimination endogène intestinale de I’ dépend de lá quantité de salive sécrétée. Cepenclant, cette perte est supérieure an strict besoin d'entretien, estimé à 2.5-30 mg de I’ par kg et par jour pour ce type de régine.

En conséquence, il est recommandé de fournir à des agneaux de 30 kg nourris de fourrages grossiers et gagnant $150 \mathrm{~g}$ par jour, des apports journaliers de $5,5 \mathrm{k}$ de calcium et de $2,2 \mathrm{~g}$ de phosphore, c'est-à-clire dans un rapport de 2,5 environ.

\section{IN'TRODUCTION}

Dans le calcul des recommandations minérales alimentaires par la méthode factorielle, l'adoption de valeurs moyennes pour les coefficients d'utilisation digestive réelle (CUI)r) demeure l'une des principales difficultés, surtout pour le calcium et le phosphore. En effet, le CUI)r de ces éléments minéraux peut varier considérablement en fonction de la nature du régime ou de la quantité ingérée. 
Les données disponibles sur l'utilisation digestive réelle du calcium et du phosphore chez le Mouton en croissance sont relativement rares. La plupart des mesures de bilans minéraux ne permettent d'évaluer que l'absorption apparente. Les recommandations britanniques (Agricultural Research Council, I965) sont basées sur des CUDr moyens de 80 à $90 \mathrm{p}$. Ioo pour le phosphore et de $50 \mathrm{p}$. Ioo environ pour le calcium chez les agneaux en croissance. Cependant, ces valeurs résultaient d'un faible nombre d'essais effectués à l'aide de méthodes radioisotopiques. Ėlles doivent être révisées.

Depuis I964, plusieurs publications portant sur le métabolisme phospho-calcique chez le Mouton en croissance ont permis de montrer que les valeurs de CUI)r adoptées clans le rapport A.R.C. étaient surestimées, notamment pour le calcium (Preston et Píander, I964; Young et al., rg66 $a$ et $b$; Compìre, ig67 ; SaintLaurent, i 970 ; Braithwaite et Riazuddin, i97 ; Madison et al., i97 I ; GueGUEN et BFSANÇON, I97I ; BRAITHWAITE, I975; GUEGUEN it al., I976). Malgré les précisions ainsi obtenues, de grandes incertitudes subsistent encore. In effet, la plupart des travaux cités portent sur des rations mixtes, tantôt très riches en calcium, tantôt carencées en phosphore dans le but d'étudier l'utilisation d'un phosphate ajouté. Ainsi, paradoxalement, le CUI)r du phosphore et du calcium est mieux connu dans le cas des sels minéraux complémentaires que dans celui des aliments simples de la ration de base.

Il nous a donc semblé intéressant d'étudier chez l'Agneau l'utilisation des principaux éléments minéraux d'une ration simple, l'ensilage de maïs supplémenté en urée mais sans adjonction de complément minéral vitaminé. Ires apports phosphocalciques sont ainsi relativement faibles et bien équilibrés, ce qui est favorable à une bonne utilisation digestive des deux éléments. De plus, les pertes fécales endogènes obtenues dans ces conditions alimentaires doivent permettre de préciser le besoin net d'entretien en phosphore et de discuter sa signification.

\section{MATÉRIEI ET MÉTHODES}

\section{1. - Animaux et alimentation}

L'essai a porté sur 6 agneaus de race lle-de-France pesant en moyenne $27,9 \quad \ldots . .0,6 \mathrm{~kg}$ au milieu de la période de bilans.

Les animaux, recevant préalablement un alinent complet à base de céréales et de foin de graminées, ont été progressivement adaptés à un régime d'ensilage de maïs (plante entic̀re) environ I mois avant l'expéricnce. Le maïs ensilé contenait en moyenne $3,8 \mathrm{~g}$ de calcium, $2, \mathrm{I} g \mathrm{~g}$ de phosphore, $I \mathrm{I},+\mathrm{g}$ le potassium, $0,25 \mathrm{~g}$ de sodium, I, $3 \mathrm{~g}$ cle magnésium et $85 \mathrm{~g}$ de matières azotées totales $(\mathrm{N} \times 6,25)$ par $\mathrm{kg}$ de nuatière sèche. Un supplément d'uréc était distribué à rai:on de I p. Ioo de la matière sèche offerte, étevant ainsi la teneur en matières ‘lzotées à I I, p. roo de la matière sèche. Durant la période expérimentale, les quantités consommées ont été limitées afin de minimiser les refus et ont été en moyenne par animal de $2,3 \mathrm{ks}$ d'ensilage frais contenant 35,7 p. Ioo de matière sèche.

\section{2. - Déroulement de l'oxpérience}

Après adaptation au régime expérimental, les quantités consommées ont été maintenues constantes durant la période de bilans.

Une semaine après une injection sous-cutanée d'environ $2 \mathrm{mCi}$ de ${ }^{32} \mathrm{I}^{2}$ sous forme de phosphate disodique, des bilans minéraux et azoté ont été effectués pendant ro jours. Les fèces et l'urine étaient récoltées séparément ct pesées. Des échantillons représentatifs de fèces, d'urine, 
d'aliment et cle refus étaient prélevés chaque jour pour analyse. Des prélèvements de sang ont été effectués dans la veine jugulaire le $7^{\mathrm{c}}$, le $10^{\mathrm{e}}$ ot le $13^{\mathrm{e}}$ jour après l'injection de ${ }^{3:{ }^{p}}$ pour déterminer la radioactivité spécifique du phosphore du plasma.

\section{3. - Méthodes de dosage ct calculs}

Les échantillons d'aliment, de refus, de fèces, d'urine et de plasma ont été séchés puis minćralisés au four à $550^{\circ} \mathrm{C}$. Après dissolution acide des cendres ct dilution convenable, les dosages chimiques ont été effectués par colorimétric au molybdovanadate d'ammonium pour le phosphore, par photométrie d'émission de flamme pour le sodium, le potassium et le calcium (photomètre Eppendorf) et par spectrophotométric d'absorption atomique pour le magnésium (spectrophotomètre IL I 5I). Les mesures de radioactivité sur les solutions aqueuses d'urine, de plasma et de fèces minéralisés ont été réalisées à l'aide d'un compteur à scintillation (Nuclear-Chicago) en utilisant l'effet Ccrenkov.

Les valeurs de la radioactivité spécifique du phosphore du plasma cntre le $7^{\text {te }}$ ct le $3^{\mathrm{e}}$ jour et des fèces entre le $8 \mathrm{e}$ et le $\mathrm{I} \mathrm{f}^{\mathrm{c}}$ jour après l'injection de ${ }^{32} \mathrm{P}$ ont permis de calculer les pertes enclogènes fécales de phosphore à l'aide de la méthode de dilution isotopique que nous avons déjà décrite (Gueguex, I962).

Le dosage de l'azote dans l'aliment et les fèces a été effectué par la méthode Kjeldahl après minéralisation sulfurique.

\section{RÉSULTATS}

Après quelques difficultés d'adaptation, les agneaux ont bien accepté le régime d'ensilage de maìs seul et ont consommé régulièrement une quantité moyenne journalière de $830 \mathrm{~g}$ de matière sèche. Cet apport alimentaire a permis un gain moyen de $\mathrm{I} 4 \mathrm{O} \mathrm{g}$ par jour durant la période expérimentale, ce qui est relativement faible par rapport au gain obtenu avec des régimes plus complets. L'utilisation digestive de la matière sèche de cette ration a été de $69, \mathrm{I} \pm 0,5 \mathrm{p}$. Ioo et celle de la matière azotée de $64,4+\mathrm{I}, \mathrm{O}$ p. I00. Les bilans azotés ont été positifs, soit en moyenne

\section{TABIEAU I}

Bilans du phosphore et du calcium

che: des agneaux Ile-de-France recerant une ration d'ensilage de mais et d'uré
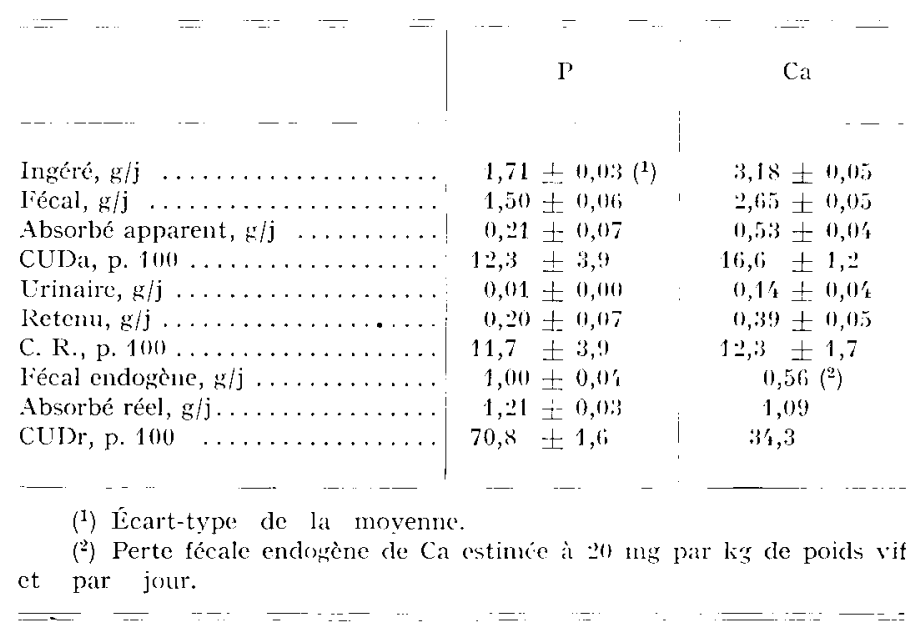
4,0 $\pm 0,4 \mathrm{~g}$ par jour, valeur relativement faible mais compatible arec le gain de poids enregistré.

Les résultats de bilans minéraux sont présentés dans les tableaux I et 2 .

TABLEAU 2

Bilans du potassium, du sodium ct du magnésium

chez des agneaux Ile-de-l'rance recevant une ration d'nsilage de maïs et d'urée'

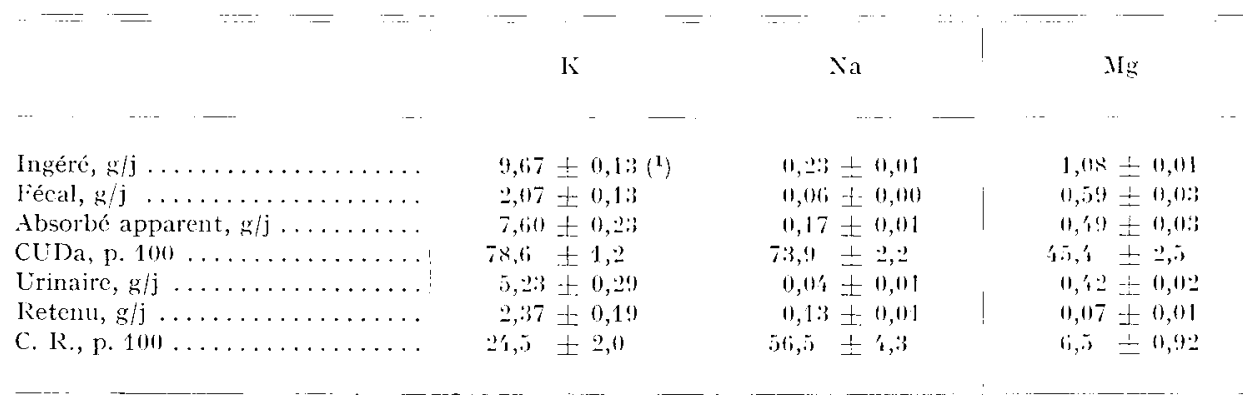

(1) Ecart-type de la moyeme.

I e phosphore du maïs ensilé est bien absorbé puisque le coefficient d'utilisation digestive réelle (CUDr) s'élève à $7 \mathrm{I}$ p. Ioo, alors que le CUD apparent (CUDa) n'atteint que I2 p. Ioo. Cet écart résulte du fait que $66 \mathrm{p}$. I oo du phosphore fécal sont d'origine endogène. L'élimination urinaire de $\mathrm{P}$ étant négligeable, la rétention est égale à l'absorption apparente, ce qui est très courant chez les ruminants.

Nous avons adopté, pour le calcium, une valeur constante de $25 \mathrm{mg}$ par $\mathrm{kg}$ de poids rif et par jour pour évaluer les pertes endogènes totales, très peu variables chez les ruminants (Gumguen, I973). Puisque l'élimination urinaire de Ca s'élève à $5 \mathrm{mg}$ par $\mathrm{kg}$ et par jour, la perte fécale endogène est de $20 \mathrm{mg}$ par $\mathrm{kg}$ et par jour. Compte tenu de cette valeur moyenne, le CLDr du calcium est de 34 p. roo, pour un CUDa de 6,6 p. Ioo.

Les quantités retenues de $\mathrm{Ca}$ et de $\mathrm{P}$ sont respectivement de 0,39 et de $0,20 \mathrm{~g}$ par jour et se situent donc dans un rapport proche de 2 .

Pour ce qui concerne le sodium, le potassium et le magnésium, nous disposons seulement des bilans apparents, ayant renoncé à adopter des valeurs théoriques moyennes pour les pertes fécales endogènes. Il apparait cependant que l'utilisation digestive du potassium et du sodium est très élevée et que la perte urinaire de sodium est négligeable. Le magnésium est également bien absorbé mais, compte tenu des pertes urinaires, sa rétention est faible.

\section{DISCUSSION}

Malgré la vitesse de croissance relativement faible permise par le régime d'ensilage de maîs enrichi en urée (I $40 \mathrm{~g}$ par jour), il s'avère que les apports alimentaires de calcium et de phosphore ainsi assurés étaient très nettement insuffisants. Fn effet, 
la rétention journalière de calcium est de $0,4 \mathrm{~g}$ seulement alors que, selon les données connues (Agricultural Research Council, I965) elle devrait être de l'ordre de I,3 g, soit 3 fois plus. De même, la rétention journalière de phosphore devrait être triplée pour assurer une bonne minéralisation osseuse. Il est donc évident, ce qui est bien connu, que le maïs seul ne permet pas de couvrir les besoins phospho-calciques des moutons en croissance, même poutr des gains de poids faibles.

Cet apport alimentaire faible était donc favorable à l'efficacité de l'utilisation digestive de ces deux éléments. İn fait, le phosphore du maïs est très bien absorbé puisque son CUDr atteint 70 p. roo comme celui du phosphore des meilleurs phosphates minéraux (Preston et PFANder, I964; Lörcher ct al., I965; CoMPÈre, I969; GuEGUEx et al., I976). Nos données confirment aussi les deux valeurs de 66 et 70 p. IOO obtenues par CoMrèrE (I967) pour le CUI)r du phosphore du maïs ensilé chez deux moutons en fin de croissance et elles montrent à nouveau que l'agneau utilise très bien le phosphore des fourrages.

Par contre, malgré l'apport alimentaire très modéré, le calcium du maìs ensilé est faiblement absorbé, puisque son CUDr ne dépasse pas $34 \mathrm{p}$. Ioo. Il est cependant possible qu'un supplément de vitamine $\mathrm{D}$ aturait permis une meilleure utilisation digestive du calcium. Nous avons déjà insisté sur la faible efficacité de l'absorption intestinale du calcium chez les ruminants (GUeguen et BEsAnçon, I97I ; GuEGUEN, I973; GUEGUEN et al., I976) et avons adopté pour établir des recommandations alimentaires des valeurs moyennes pour le CUDr diminuant de 35 p. I00 à $30 \mathrm{~kg}$ à $25 \mathrm{p}$. Ioo seulement à $50 \mathrm{~kg}$. Cela est en parfait accord avec les données obtenutes dans les mêmes conditions par Young et al. (I966), BraithwaITE et Riazuddin (I97I) et BRAithwaIte (I975).

Compte tenu des pertes fécales endogènes, le potassium et le sodium du maïs sont presque totalement absorbés et le CUDr du magnésium dépasse $50 \mathrm{p}$. Ioo. Par comparaison aux données connues (A.R.C., I965), la rétention journalière est normale pour le magnésium, trop faible pour le sodium dont l'apport alimentaire est insuffisant et trop forte pour le potassium, ce que nous ne pouvons expliquer.

La parte endogène fécale de $36 \mathrm{mg}$ de phosphore par $\mathrm{kg}$ de poids vif et par jour, que nous avons obtenue, concorde parfaitement avec les données déjà citées pour des moutons en croissance recevant des régimes similaires (COMPÈre, I967; ANNENKov et al., I97 I a). Flle diffère peu de la valeur moyenne de $40 \mathrm{mg}$ adoptée par 1'A.R.C. (I965). La variabilité de l'excrétion fécale endogène de phosphore chez les ruminants est bien connue et les résultats cités varient entre Io et $60 \mathrm{mg}$ par $\mathrm{kg}$ et par jour. Les valeurs les plus faibles ont été enregistrées chez des brebis en fin de gestation consommant des régimes concentrés ou seni-synthétiques assez pauvres en phosphore (ANinenkov et al., I97 i $b$; Sykes et Dingwaid, I976) tandis que les valeurs les plus élevées concernent des moutons en croissance recevant des régimes riches en phosphore et à base de fourrages grossiers (BRÜGGEMANn et al., I959; Shroder et Haxsard, ig68; Gueguen et al., i976). Puisque la salive constitue chez les ruminants le principal moyen de régulation homéostatique pour le phosphore (Tomas, I974), il est évident que l'intensité du flot salivaire, qui varie suivant la nature physique de la ration, détermine le seuil minimum des pertes métaboliques inévitables de phosphore dans les fèces. Le strict besoin net d'entretien serait ainsi d'autant plus faible que les animaux consomment des régimes concentrés pauvres 
en cellulose, ce qui a déjà été mis en évidence chez le Veau (GUnGUrs, I963) et chez le Mouton (CoMpìne, I967).

De plus, pour un même type de ration de base l'excrétion fécale endogène augmente avec 1'ingestion de phosphore. Les pertes métaboliques inévitables résultant d'une sécrétion salivaire compatible avec un bon fonctionnement du rumen sont donc, en général, inférieures aux pertes fécales endogènes, dont une partie peut correspondre à une véritable excrétion d'un excès de phosphore circulant (Gueguis, I965; Compère, I967). Aussi, même pour un apport alimentaire faible qui ne permet pas une rétention osseuse normale, un certain excès de phosphore circulant peut résulter d'une insuffisance relative en calcium (seulement I, I $\mathrm{g}$ de $\mathrm{Ca}$ est absorbé contre $\mathrm{I}, 2 \mathrm{~g}$ de $\mathrm{P}$ ) qui ne permet pas l'accrétion osseuse maximale du phosphore absorbé. Il semble donc logique d'adopter une valeur plus faible, de 25 à 3o mg par kg et par jour, pour les pertes fécales correspondant au besoin net d'entretien. Cela est d'ailleurs conforme aux conclusions de Compìre (I967) qui propose un seuil minimum de $26 \mathrm{mg}$ de $P$ par $\mathrm{kg}$ et par jour et à la valeur de $24 \mathrm{mg}$ que nous avions obtenue chez des moutons adultes en bilan négatif et consommant un excès de calcium (GUEGUEN, I962).

En conclusion, les données précédentes nous conduisent à recommander un apport journalier de $5,5 \mathrm{~g}$ de calcium pour des moutons de $30 \mathrm{~kg}$ gagnant environ I50 g par jour. L'apport correspondant de phosphore, dans le cas de régimes à base de fourrages grossiers éventuellement enrichis en phosphates minéraux de bonne qualité, serait de 2,2 g environ, ce qui concorde avec les conclusions de Fishwick et Hemixgway (1973). Nous aboutissons ainsi à des besoins alimentaires de calcium et de phosphore qui se situent dans un rapport voisin de 2,5 . Un rapport phosphocalcique plus élevé, de l'ordre de 3, devrait être recommandé dans le cas de régimes plus concentrés, lorsque le besoin d'entretien en phosphore est plus faible (SYkEs et Dingwai, L, I976).

Un tel équilibre entre les deux éléments peut permettre, dans certains cas, de diminuer la phosphaturie et de prévenir l'urolithiase.

$$
\text { Reçu pour publication en juin } 1976 .
$$

\section{SUMMARY}

\section{UTILISATION OF THE ESSLNTIAL MINERALS OF MAIZE SILAGE BY GROWING SHEEP}

Vineral balances ( $\mathrm{P}, \mathrm{Ca}, \mathrm{Mg}, \mathrm{K}, \mathrm{Na}$ ) were carried out in six $28 \mathrm{~kg}$ lambs ferl maize silage

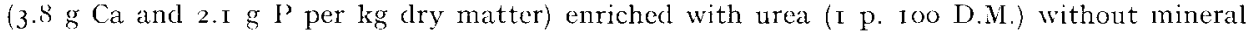
supplementation. Endogenous faecal losses of phosphorus were determined by the isotopic dilution method.

The true absorption of phosphorus reached $70 \mathrm{p}$. roo of the intake whereas only $3+p .100$ calcium were absorbed (table 1 ).

The daily balances were $0.39 \mathrm{~g}$ for calcium and $0.20 \mathrm{~g}$ for phosphorus, these values representing about one third of the requirements for a daily weight gain of yo g. Likewise, the sodium supply was insufficient.

Endogenous faecal losses of phosphorus represented $06 \mathrm{p}$. Ioo of total faecal losses, i.e. $36 \mathrm{mg}$ per $\mathrm{kg}$ live weight and per day. The latter value is normal for a diet based on roughage, as endogenous intestinal secretion of $\mathrm{P}$ depends on the amount of saliva. However, this loss is higher than the strict maintenance requirement estimated at 25-30 $\mathrm{mg} \mathrm{P}$ per $\mathrm{kg}$ and per day with this type of dict.

Consequently, for $30 \mathrm{~kg}$ lambs fed with roughage and gaining $150 \mathrm{~g}$ per day a daily supply of $5.5 \mathrm{~g}$ calcium and $2.2 \mathrm{~g}$ phosphorus, $i, c$ at a ratio of about 2.5 is arlvisable. 


\section{RÉFÉRENCES BIBLIOGRAPHIQUES}

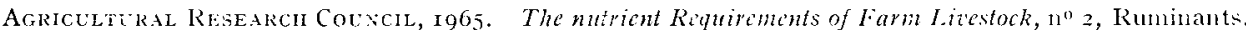
I. M.S.O, London.

AnNingov 13. N., Manisox V. 1., Lomonix N. G., I97 a. Calcium and phosphorus metabolism in sheep (ell ruste). Sel'skohoz. Biol., 6, 85-90.

Annenkov B. X., Madison V. I., Iobodix N. (i., I)mozdesko A. I)., I97 $b$. Assimilation and metabolism of phosphorus in pregnant and lactating ewes given different anounts of calcium and nhosphorus in the feed (en russe). Doklady Tsesoyuznoi ordena Lenina Akad. Sel' skohoz. Nauki, 8, 35-36.

Bratthwate (i. D., I975. Studies on the absorption and retention of calcitin and phosphorus by young and wnature Ca-deficient sheep. IBr.J. Nutr., 34, 3 I I-324.

J3RaIthwatt: (;. I)., Riazubur Sh., I97I. The effect of age and level of dietary calcium intake on calciun metabolism in sheep. Br.J. Nutr., 26, 2 I 5-225.

Bröggemaxi J., Bronsch K., lörchlir K., Sluss F., I959. Die J3estimmung det Phosphorresorption aus Mineralfuttern. Z. Tierphys. Tiercrnähr. Futtermitt., 14, 224-24I.

Comprire lR., I967. L'étude de l'équilibre phospho-calcjque du régime bascé sur les variations du métabolisme phosphoré. Les Presses Agronomiques de Gemblonx, ćl. Duculot, $\mathrm{S}$. A.

Compíre R., I969. Valeur comparée des phosphates monoamnonique et diammonique comme sources de phosphore et d'azote alimentaire chez le Mouton. Bull. Rech. Agron., Gembloux, tome IV, 339-367.

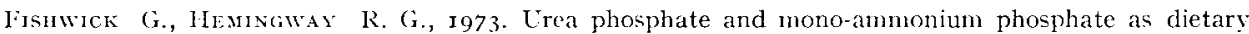
supplements for sheep fed diets inadequate in phosphorus and nitrogen. J. Agric. Sci., 81, I39-I43.

GIEGEX L., I962. J'utilisation digestive réclle du phosphore du foin de luzerne par le Mouton, mesurée à l'aide de ${ }^{32 P}$. Ann. Biol. anim. Bioch. Biophys., 2, i 43 -I 49.

Guequen L., I963. Influence de la nature du régime alimentaire sur l'excrétion fécale de phosphore endogène chez le veau. Ann. Biol. anim. Bioch, Biophys., 3, 243-253.

Giciuen I., I965. Contribution à l'étude de l'absorption intestinale et du mode d'excrétion clu phosphore chez les aninalux. Meded. Landbouth. Opzoek. Cient., 30, 687-697.

GLegrex 1., Besascos P., r972. Influence des sulfates sur le métabolisme phospho-calcique. I. Utilisation du sulfate de calcium par le Mouton. Ann. Biol. anim. Bioch. Biophys., 12, 589-598.

Greces L., I973. Ie métabolisme du calcium chez les ruminants. In : Physiologie comparée des lichange's calciques $"$ D. Patusu, Simep éd., 69 Villeurbanne, $67-78$.

Griciven L., loret R., Dtrand Michelle, i976. Utilisation du phosphate mono-ammonique chez le Mouton. I. Itilisation comparce du phosphore des phosphates niono-ammonique et monosodique et infuence sur le métabolisme du calciun et du magnésium. Ann. Zootech., 25, I I I-I I8.

Lörcher K, Broxsci K., Coxrad C., I965. Resorptionsstudien an Nutztieren 5; Die Phosphorresorption bein Schaf nach Verfütterung ejner mit verschiedenen Phosphaten supplenentierten Standard Ration. Z. Tierphysiol. Tierernähr. Futtermitt., 20, 234-24.3.

Mabisox I. L., Axwexkov B, N., Fomicer Ju. I', r97I. Radioisotope nethods for studying assimilation of phosphorus from fodder phosphates by sheep. l'estn. Selskohoz. Nauki, 2, ri6-i i 9.

PREsTON R. L., PFAnder W. I., I964. l'hosphorus metabolism in lanb; fed varying phosphorus intalies. J. Nutr., 83, 369-378.

SHRODER J. I), HANsARD S. I., I958. Effects of orally administered stilbestrol upon growth and upon calcium and phosphorus netabolisin in lambs. J. anim. Sci., 17, 343-352.

Saixt-Lavrext (i. J., I970. Calcium nutrition of the lamb: Part I. Calcium and phosphorus recuirements. Part II. Metabolism of calcium. Ph. D. Dissert. Cornell L'niversity.

Sykes A. R., 1)ixgwal R. A., I976. The phosphorus requirenent of pregnant sheep. $J$. agric. Sci, $83,587-504$.

Tomas F. M., I974. Phosphorus homeostasis in sheep. III. Relationship between the anount of salivary phosphorus secreted and the quantities of phosphorus excreted via the urine and faeces. $A u s t r . J$. Agric. K'es., 25, 495-507.

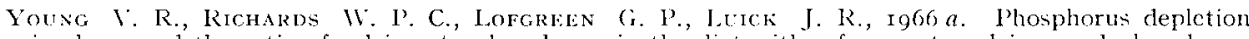
in sheep and the ratio of calcium to phosphorus in the diet with reference to calcium and phosphorus absorption. Br. J. Nutr., 20, $783-794$.

Young V. R., LoFgrielis (i. P., Luick J. R., I966 b. The effects of phosphorus depletion, and of calcium and phosphorus intake, on the endogenous excretion of these elements by sheep. Br. J. Natr., 20, 79.5 .805 . 